\title{
ON THE NUMERICAL SOLUTION \\ OF THE ONE-DIMENSIONAL CONVECTION-DIFFUSION EQUATION
}

\author{
MEHDI DEHGHAN
}

Received 20 March 2004 and in revised form 8 July 2004

The numerical solution of convection-diffusion transport problems arises in many important applications in science and engineering. These problems occur in many applications such as in the transport of air and ground water pollutants, oil reservoir flow, in the modeling of semiconductors, and so forth. This paper describes several finite difference schemes for solving the one-dimensional convection-diffusion equation with constant coefficients. In this research the use of modified equivalent partial differential equation (MEPDE) as a means of estimating the order of accuracy of a given finite difference technique is emphasized. This approach can unify the deduction of arbitrary techniques for the numerical solution of convection-diffusion equation. It is also used to develop new methods of high accuracy. This approach allows simple comparison of the errors associated with the partial differential equation. Various difference approximations are derived for the one-dimensional constant coefficient convection-diffusion equation. The results of a numerical experiment are provided, to verify the efficiency of the designed new algorithms. The paper ends with a concluding remark.

\section{Introduction}

The behavior of fluid undergoing mass, vorticity, or forced heat transfer is described by a set of partial differential equations which are mathematical formulations of one or more of the conservation laws of physics. These laws include those of conservation of mass, momentum, and energy. For instance, if the fluids are incompressible with a density which is independent of temperature and has constant thermal conductivity $k$, the heat equation, which is a mathematical formulation of the law of conservation of thermal energy in the absence of sources of sinks, governs the distribution of temperature $u$.

This equation is

$$
\frac{\partial u}{\partial t}+a \frac{\partial u}{\partial x}+b \frac{\partial u}{\partial y}+c \frac{\partial u}{\partial z}-\alpha\left(\frac{\partial^{2} u}{\partial x^{2}}+\frac{\partial^{2} u}{\partial y^{2}}+\frac{\partial^{2} u}{\partial z^{2}}\right)=0,
$$

in which $\alpha=k / p D_{p}$ and $a, b, c$ are velocity components of the fluid in the directions of the axes at the point $(x, y, z)$ at time $t$. Note that $p$ is the density, $D_{p}$ is the specific heat 
of the fluid at constant pressure, and $\partial / \partial t$ is the operation of differentiation following the motion of the fluid. For fluids at rest, and for solids, $a=b=c=0$. In this case, (1.1) reduces to the pure diffusion equation

$$
\frac{\partial u}{\partial t}-\alpha\left(\frac{\partial^{2} u}{\partial x^{2}}+\frac{\partial^{2} u}{\partial y^{2}}+\frac{\partial^{2} u}{\partial z^{2}}\right)=0
$$

Equation (1.1) is a form of the so-called convection-diffusion equation; heat is carried along with the moving fluid (convection) and spreads due to diffusion (conduction). The three terms $a(\partial u / \partial x), b(\partial u / \partial y)$, and $c(\partial u / \partial z)$ are usually called convective (or sometimes advective) terms, and the three terms $\alpha\left(\partial^{2} u / \partial x^{2}\right), \alpha\left(\partial^{2} u / \partial y^{2}\right)$, and $\alpha\left(\partial^{2} u / \partial z^{2}\right)$ are usually called diffusive (or sometimes viscous) terms.

In this paper, we will consider the one-dimensional convection-diffusion equation

$$
\frac{\partial u}{\partial t}+a \frac{\partial u}{\partial x}=\alpha \frac{\partial^{2} u}{\partial x^{2}}, \quad 0<x<1,0<t \leq T
$$

with initial condition

$$
u(x, 0)=f(x), \quad 0 \leq x \leq 1
$$

and boundary conditions

$$
\begin{array}{ll}
u(0, t)=g_{0}(t), & 0<t \leq T, \\
u(1, t)=g_{1}(t), & 0<t \leq T,
\end{array}
$$

where $f, g_{0}$, and $g_{1}$ are known functions, while the function $u$ is unknown. Note that $\alpha>0$ and $a>0$ are considered to be positive constants quantifying the diffusion and advection processes, respectively.

For more applications of the convection-diffusion equation see $[1,2,3,4,6,7,8,9,10$, $11,12,13,14,16,17,18,19,22]$.

Little progress has been made toward finding analytical solutions to the one-dimensional convection-diffusion equation even with $\alpha$ and $a$ constant, when initial and boundary conditions are complicated. A few special cases have been reported [19], such as initial condition $u(x, 0)=0,0 \leq x \leq 1$, and boundary values $u(0, t)=1$ and $u(1, t)=0$, $0<t \leq T$. Consequently, much effort has been put into developing stable and accurate numerical solutions of (1.3). Numerical techniques for (1.3) are by now well developed and many useful schemes have been established such as finite differences, finite elements, spectral procedures, the method of lines, and so forth. Finite difference techniques for solving the one-dimensional convection-diffusion equation can be considered according to the number of spatial grid points involved, the number of time-levels used, whether they are explicit or implicit in nature. 
This paper contains a new approach. We have proposed a new practical schemedesigning approach whose application is based on the modified equivalent partial differential equation (MEPDE). This approach can unify the deduction of arbitrary schemes for the solution of the convection-diffusion equation in one-space variable. This approach is especially efficient in the design of higher-order techniques.It allows the simple determination of the theoretical order of accuracy, thus allowing methods to be compared with one another. Also from the truncation error of the modified equivalent equation, it is possible to eliminate the dominant error terms associated with the finite difference equations that contain free parameters, thus leading to more accurate methods.

In the present research employing the new approach, various numerical finite difference schemes will be developed and compared for solving this equation. We consider methods that are second-order accurate and techniques that are third-order or fourthorder accurate.

A major issue in numerical algorithms used to solve partial differential equations, like the convection-diffusion equation, is stability. To analyze the stability of the developed schemes, the amplification factor for a Fourier method in space is determined.

We will now summarize the remainder of the paper. Several two-level finite difference methods for the solution of (1.3)-(1.5) are given in Section 2. Notations appear in this section. The accuracy and efficiency of the presented procedures are also described in Section 3. Section 3 contains a discussion of the stability and accuracy of approximations in one-space dimension with Dirichlet's boundary conditions. The results of a numerical experiment are presented in Section 4 to demonstrate the efficiency of the discussed algorithms. Some concluding remarks and suggestions for future research are outlined in Section 4.

\section{The finite difference schemes}

Like many other numerical approaches, our approach begins with a discretization of the domain of the independent variables $x$ and $t$.

We subdivide the interval $[0,1]$ into $M$ subintervals such that $M h=1$ and the interval $[0, T]$ into $N$ subintervals such that $N l=T$. We can then approximate $u(x, t)$ by $u_{i}^{n}$, value of the difference approximation of $u(x, t)$ at the point $x=i h$ and $t=n l$, where $0 \leq i \leq M$ and $0 \leq n \leq N$.

Hence the grid points $\left(x_{i}, t_{n}\right)$ are defined by

$$
\begin{aligned}
& x_{i}=i h, \quad i=0,1,2, \ldots, M, \\
& t_{n}=n l, \quad n=0,1,2, \ldots, N,
\end{aligned}
$$

in which $M$ and $N$ are integers.

2.1. Second-order upwind explicit technique. Using (1.3) we have

$$
\left.\frac{\partial u}{\partial t}\right|_{i} ^{n}+\left.\left.a \frac{\partial u}{\partial x}\right|_{i} ^{n} \simeq \alpha \frac{\partial^{2} u}{\partial x^{2}}\right|_{i} ^{n} .
$$


This explicit technique uses the following approximations for $\partial u / \partial t, \partial u / \partial x$, and $\partial^{2} u /$ $\partial x^{2}$, respectively,

$$
\begin{gathered}
\left.\frac{\partial u}{\partial t}\right|_{i} ^{n} \simeq \frac{\left(u_{i}^{n+1}-u_{i}^{n}\right)}{\Delta t}, \\
\left.\frac{\partial u}{\partial x}\right|_{i} ^{n} \simeq \frac{4 s-2 c+3 c^{2}}{4 c} \frac{\left(u_{i}^{n}-u_{i-2}^{n}\right)}{2 \Delta x}+\frac{4 s-2 c+c^{2}}{4 c} \frac{\left(u_{i+2}^{n}-u_{i}^{n}\right)}{2 \Delta x} \\
+\frac{2 c-c^{2}-2 s}{c} \frac{\left(u_{i+1}^{n}-u_{i-1}^{n}\right)}{2 \Delta x}, \\
\left.\frac{\partial^{2} u}{\partial x^{2}}\right|_{i} ^{n} \simeq \frac{2 c-c^{2}-2 s}{2 s} \frac{\left(u_{i+1}^{n}-2 u_{i}^{n}+u_{i-1}^{n}\right)}{(\Delta x)^{2}}+\frac{4 s-2 c+c^{2}}{2 s} \frac{\left(u_{i+2}^{n}-2 u_{i}^{n}+u_{i-2}^{n}\right)}{(2 \Delta x)^{2}} .
\end{gathered}
$$

Putting the above approximations into (2.3) yields the following finite difference equation:

$$
u_{i}^{n+1}=\frac{1}{2}\left(2 s-c+c^{2}\right) u_{i-2}^{n}-\left(2 s-2 c+c^{2}\right) u_{i-1}^{n}+\frac{1}{2}\left(2+2 s-3 c+c^{2}\right) u_{i}^{n}
$$

for $1 \leq i \leq M-1$ and $0 \leq n \leq N-1$, where

$$
\begin{gathered}
c=a \frac{\Delta t}{\Delta x}, \\
s=\alpha \frac{\Delta t}{(\Delta x)^{2}} .
\end{gathered}
$$

In order to determine the criteria for (2.7) to be von Neumann stable [20] we consider the amplification factor $G$ :

$$
G=\frac{1}{2}\left(2 s-c+c^{2}\right) \exp (-2 i \beta)+\left(-2 s+2 c-c^{2}\right) \exp (-i \beta)+\frac{1}{2}\left(2+2 s-3 c+c^{2}\right) .
$$

For stability, it is required that

$$
|G| \leq 1 \text {. }
$$

It can be easily seen that (2.7) is stable for values of $c$ and $s$ which satisfy

$$
0<s \leq \frac{c(2-c)}{2} .
$$

Note that it is twice the size of the corresponding stability region for the Lax-Wendroff method.

Note that a numerical method must be stable, in order to be implemented on a computer. However, this is only one of the desirable properties of a numerical solution of a partial differential equation. Others include that it should be convergent, that is should be accurate and that it should realistically reflect special properties of the solution of the given partial differential equation. For example, in the convection-diffusion equation which models the spread of contaminants in fluids, prediction of negative values in concentration is physically unrealistic. 


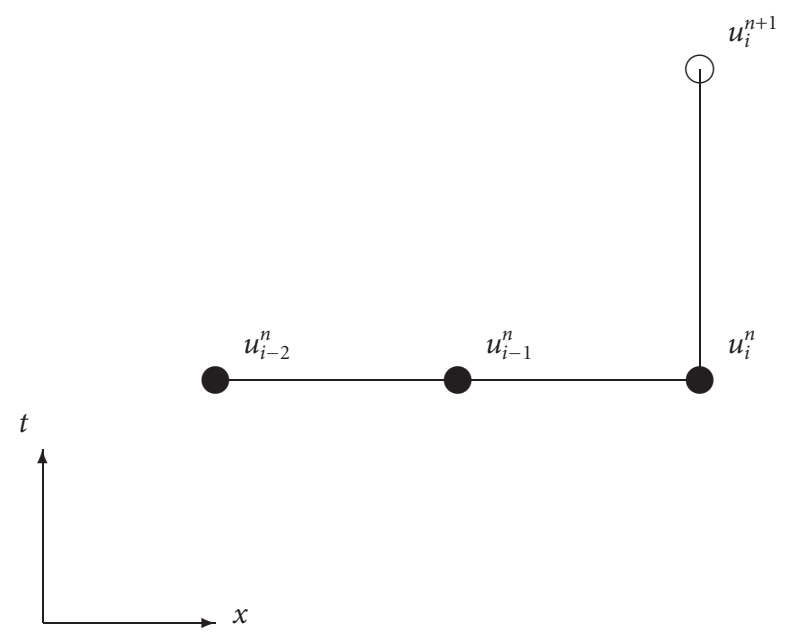

Figure 2.1. Computational molecule for the $(1,3)$ upwind formula.

The MEPDE [21] of this method is in the following form:

$$
\frac{\partial u}{\partial t}+a \frac{\partial u}{\partial x}-\alpha \frac{\partial^{2} u}{\partial x^{2}}+\frac{c(\Delta x)^{2}}{6} \frac{\left(6 s-2 c+c^{2}-6 s c+2 c^{2}-c^{3}\right)}{c} \frac{\partial^{3} u}{\partial x^{3}}+O\left((\Delta x)^{3}\right)=0 .
$$

It can be easily seen that the wave speed error is minimal when either

$$
c=1
$$

or

$$
s=\frac{c(2-c)}{6} .
$$

The computational molecule of this formula is shown in Figure 2.1. In the following the procedure using this formula will be referred to as the $(1,3)$ method, because the molecule involves one grid point at the new time-level and 3 at the old level.

2.2. Third-order upwind explicit technique. This technique uses (2.4) for approximating the time derivative and uses the following approximations for spatial derivatives:

$$
\begin{aligned}
\left.\frac{\partial u}{\partial x}\right|_{i} ^{n} \simeq & \frac{2 c^{2}+3 c+12 s-2}{12} \frac{\left(u_{i}^{n}-u_{i-2}^{n}\right)}{2 \Delta x}+\frac{2 c^{2}-3 c+12 s-2}{12} \frac{\left(u_{i+2}^{n}-u_{i}^{n}\right)}{2 \Delta x} \\
+ & \frac{4-c^{2}-6 s}{3} \frac{\left(u_{i+1}^{n}-u_{i-1}^{n}\right)}{2 \Delta x}, \\
\left.\frac{\partial^{2} u}{\partial x^{2}}\right|_{i} ^{n} \simeq & \frac{6 s-12 s c+2 c-2 c^{3}+3 c^{2}}{6 s} \frac{\left(u_{i+1}^{n}-2 u_{i}^{n}+u_{i-1}^{n}\right)}{(\Delta x)^{2}} \\
& +\frac{12 s c-2 c+2 c^{3}-3 c^{2}}{6 s} \frac{\left(u_{i+2}^{n}-2 u_{i}^{n}+u_{i-2}^{n}\right)}{2(\Delta x)^{2}} .
\end{aligned}
$$




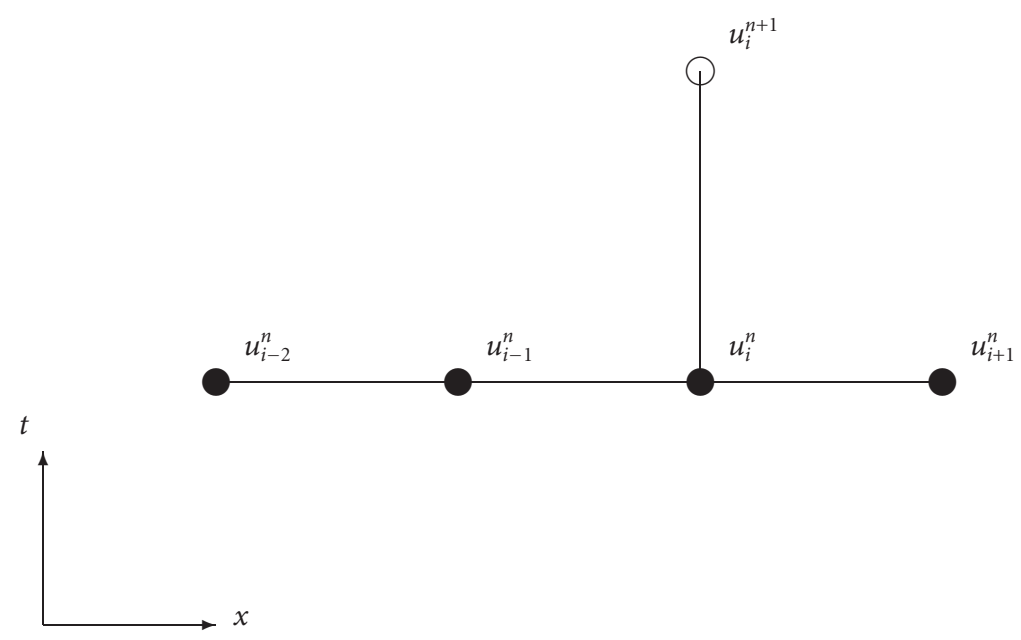

Figure 2.2. Computational molecule for the $(1,4)$ upwind formula. tion:

Putting the above approximations into (2.3) yields the following finite difference equa-

$$
\begin{aligned}
u_{i}^{n+1}= & \frac{1}{6} c\left(c^{2}+6 s-1\right) u_{i-2}^{n}+\frac{1}{2}\left(2 s+2 c+c^{2}-c^{3}+6 s c\right) u_{i-1}^{n} \\
& +\frac{1}{2}\left(2-4 s+6 s c-c-2 c^{2}+c^{3}\right) u_{i}^{n}+\frac{1}{6}(1-c)\left(6 s-2 c+c^{2}\right) u_{i+1}^{n} .
\end{aligned}
$$

In order to determine the criteria for (2.18) to be von Neumann stable [15] we consider the von Neumann amplification factor $G$ :

$$
\begin{aligned}
G= & \frac{1}{6} c\left(c^{2}+6 s-1\right) \exp (-2 i \beta)+\frac{1}{2}\left(2 s+2 c+c^{2}-c^{3}+6 s c\right) \exp (-i \beta) \\
& +\frac{1}{2}\left(2-4 s+6 s c-c-2 c^{2}+c^{3}\right)+\frac{1}{6}(1-c)\left(6 s-2 c+c^{2}\right) \exp (i \beta) .
\end{aligned}
$$

For stability, it is required [15] that $|G| \leq 1$.

It can be easily seen that (2.18) is stable for values of $c$ and $s$ which satisfy (2.12).

The MEPDE [21] of this method is in the following form:

$$
\begin{aligned}
\frac{\partial u}{\partial t} & +a \frac{\partial u}{\partial x}-\left[\alpha+\frac{\beta \Delta x(1-c)}{2}\right] \frac{\partial^{2} u}{\partial x^{2}} \\
& +\frac{c(\Delta x)^{2}}{6} \frac{6 s-2 c+c^{2}-6 s c+2 c^{2}-c^{3}}{c} \frac{\partial^{3} u}{\partial x^{3}}+O\left((\Delta x)^{3}\right)=0 .
\end{aligned}
$$

This method has the computational molecule shown in Figure 2.2. In the following the procedure using this formula will be referred to as the $(1,4)$ method, because the molecule involves one grid point at the new time-level and 4 at the old level.

Note that this third-order method is fully explicit and can therefore be used to maximum advantage on a parallel computer. 
2.3. The fourth-order upwind explicit scheme. This procedure uses the approximation (2.4) for the derivative with respect to the time variable and uses the following weighted approximation for the second-order derivative with respect to the spatial variable:

$$
\begin{aligned}
\left.\frac{\partial^{2} u}{\partial x^{2}}\right|_{i} ^{n} \simeq & \frac{\left(-c^{4}+4 c^{2}-12 s^{2}-12 s c^{2}+8 s\right)}{6 s} \frac{\left(u_{i+1}^{n}-2 u_{i}^{n}+u_{i-1}^{n}\right)}{(\Delta x)^{2}} \\
& +\frac{\left(c^{4}-4 c^{2}+12 s^{2}+12 s c^{2}-2 s\right)}{6 s} \frac{\left(u_{i+2}^{n}-2 u_{i}^{n}+u_{i-2}^{n}\right)}{(\Delta x)^{2}} .
\end{aligned}
$$

Note that this technique uses the approximation (2.16) for the first-order derivative with respect to the space variable.

This yields the following finite difference equation:

$$
\begin{aligned}
u_{i}^{n+1}= & \frac{1}{24}\left(12 s\left(s+c^{2}\right)+2 s(6 c-1)+c(c-1)(c+1)(c+2)\right) u_{i-2}^{n} \\
& +\frac{1}{24}\left(12 s\left(s+c^{2}\right)-2 s(6 c+1)+c(c-1)(c+1)(c-2)\right) u_{i-1}^{n} \\
& -\frac{1}{6}\left(12 s\left(s+c^{2}\right)+2 s(3 c-4)+c(c-2)(c+1)(c+2)\right) u_{i}^{n} \\
& -\frac{1}{6}\left(12 s\left(s+c^{2}\right)-2 s(3 c+4)+c(c-1)(c-2)(c+2)\right) u_{i+1}^{n} \\
& +\frac{1}{4}\left(12 s\left(s+c^{2}\right)-10 s+(c-1)(c-2)(c+1)(c+2)\right) u_{i+2}^{n},
\end{aligned}
$$

which is stable for values of $c$ and $s$ satisfy

$$
0<s \leq \frac{(2-c)}{2}
$$

In order to determine the von Neumann stability [15] of (2.22) we consider the amplification factor $G$ :

$$
\begin{aligned}
G= & \frac{1}{24}\left(12 s\left(s+c^{2}\right)+2 s(6 c-1)+c(c-1)(c+1)(c+2)\right) \exp (-2 i \beta) \\
& +\frac{1}{24}\left(12 s\left(s+c^{2}\right)-2 s(6 c+1)+c(c-1)(c+1)(c-2)\right) \exp (-i \beta) \\
& -\frac{1}{6}\left(12 s\left(s+c^{2}\right)+2 s(3 c-4)+c(c-2)(c+1)(c+2)\right) \\
& -\frac{1}{6}\left(12 s\left(s+c^{2}\right)-2 s(3 c+4)+c(c-1)(c-2)(c+2)\right) \exp (i \beta) \\
& +\frac{1}{4}\left(12 s\left(s+c^{2}\right)-10 s+(c-1)(c-2)(c+1)(c+2)\right) \exp (2 i \beta) .
\end{aligned}
$$

Note that the new fourth-order formula cannot be used to compute an approximate value for $u$ at the grid point next to the boundary on each side of the solution domain. Instead, extrapolation techniques or alternative finite difference formula based on other computational molecules and of the appropriate accuracy must be used to compute them. 


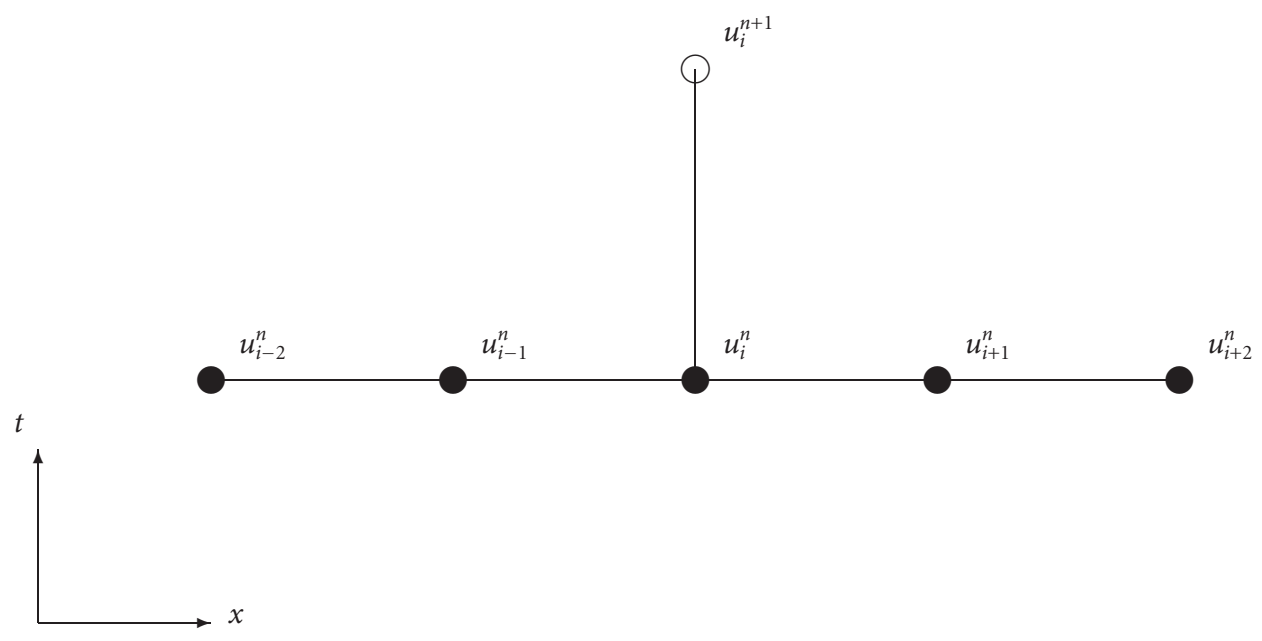

Figure 2.3. Computational molecule for the $(1,5)$ upwind formula.

The MEPDE of this method is in the following form:

$$
\frac{\partial u}{\partial t}+a \frac{\partial u}{\partial x}-\alpha \frac{\partial^{2} u}{\partial x^{2}}-\frac{a(\Delta x)^{4}\left[60 s^{2}-5 c^{2}+c^{4}-30 s+20 c s^{2}+4\right]}{120} \frac{\partial^{5} u}{\partial x^{5}}+O\left((\Delta x)^{5}\right)=0,
$$

which verifies that this technique is fourth-order accurate.

Note that the fourth-order scheme (2.22) has another advantage over the implicit formula, it is explicit and can therefore be used to maximum advantage on a vector or parallel computer.

The computational molecule of this procedure is shown in Figure 2.3. In the following the procedure using this formula will be referred to as the $(1,5)$ method, because the molecule involves one grid point at the new time-level and 5 at the old level.

Using weighted discretization with the MEPDE approach, several accurate finite difference approximations can be developed to solve the one-dimensional convection-diffusion equation.

\section{The weighted two-level explicit methods}

Convection-diffusion equations are the most common type of partial differential equations, and efficient numerical solution of such problems is of major importance in many applications. Although computer capacities are rapidly expanding, the size of the problems that are to be solved in practice easily keeps pace. The construction and analysis of numerical techniques is therefore an active field of research. The main idea behind the finite difference methods for obtaining the solution of a given partial differential equation 
is to approximate the derivatives appearing in the equation by a set of values of the function at a selected number of points. The most usual way to generate these approximations is through the use of Taylor series. The numerical techniques developed here are based on the MEPDE as described by Warming and Hyett (see [5, 21]). This approach allows the simple determination of the theoretical order of accuracy, thus allowing methods to be compared with one another. Also from the truncation error of the modified equivalent equation, it is possible to eliminate the dominant error terms associated with the finite difference equations that contain free parameters (weights), thus leading to more accurate methods.

Consider the following approximations of the derivatives in the convection-diffusion equation (1.1) which incorporate weights $\phi, \theta, \gamma$ as follows:

$$
\begin{gathered}
\left.\frac{\partial u}{\partial x}\right|_{i} ^{n} \simeq \phi \frac{\left(u_{i}^{n}-u_{i-2}^{n}\right)}{2 \Delta x}+\theta \frac{\left(u_{i+2}^{n}-u_{i}^{n}\right)}{2 \Delta x}+(1-\phi-\theta) \frac{\left(u_{i+1}^{n}-u_{i-1}^{n}\right)}{2 \Delta x} \\
\left.\frac{\partial^{2} u}{\partial x^{2}}\right|_{i} ^{n} \simeq \gamma \frac{\left(u_{i+1}^{n}-2 u_{i}^{n}+u_{i-1}^{n}\right)}{(\Delta x)^{2}}+(1-\gamma) \frac{\left(u_{i+2}^{n}-2 u_{i}^{n}+u_{i-2}^{n}\right)}{(\Delta x)^{2}}
\end{gathered}
$$

Substituting the above approximations in the convection-diffusion equation (1.1) yields the following weighted explicit finite difference formula:

$$
\begin{aligned}
u_{i}^{n+1}= & \frac{1}{4}(s+2 c \phi-s \gamma) u_{i-2}^{n}+\frac{1}{2}(c-c \phi-c \theta+2 s \gamma) u_{i-1}^{n} \\
& +\frac{1}{2}(2-s+c \theta-c \phi-3 s \gamma) u_{i}^{n}+\frac{1}{2}(-c+c \phi+c \theta+2 s \gamma) u_{i+1}^{n} \\
& +\frac{1}{4}(s-s \gamma-2 c \theta) u_{i+2}^{n},
\end{aligned}
$$

for $1 \leq i \leq M-1$ and $0 \leq n \leq N-1$, where $c$ and $s$ are defined in (2.8) and (2.9), respectively.

It can be easily seen that the corresponding amplification factor $G$ is in the following form:

$$
\begin{aligned}
G= & \frac{1}{4}(s+2 c \phi-s \gamma) \exp (-2 i \beta)+\frac{1}{2}(c-c \phi-c \theta+2 s \gamma) \exp (-i \beta) \\
& +\frac{1}{2}(2-s+c \theta-c \phi-3 s \gamma)+\frac{1}{2}(-c+c \phi+c \theta+2 s \gamma) \exp (i \beta) \\
& +\frac{1}{4}(s-s \gamma-2 c \theta) \exp (2 i \beta) .
\end{aligned}
$$

A von Neumann stability of (3.2) yields the stability condition

$$
\frac{\left(c^{2}-c \phi\right)}{2} \leq s \leq \frac{(1-c \phi)}{2} .
$$


The MEPDE of this method is in the following form [21]:

$$
\begin{aligned}
& \frac{\partial u}{\partial t}+a \frac{\partial u}{\partial x}-\left(\alpha-\frac{\beta(\Delta x)}{2}(-c+2 \phi-2 \theta)\right) \frac{\partial^{2} u}{\partial x^{2}} \\
& +\frac{a(\Delta x)^{2}}{6}\left(1-6 s+3 \theta+6 c \theta+2 c^{2}+3 \phi-6 c \phi\right) \frac{\partial^{3} u}{\partial x^{3}} \\
& +\frac{a(\Delta x)^{3}}{24 c}\left(6 s+12 c^{2} \phi^{2}+12 c^{2} \theta^{2}-24 c^{2} \theta+4 c^{2}+6 c^{4}-8 s+12 s^{2}-24 s c^{2}+24 s \phi\right. \\
& \left.\quad-8 \phi+12 c^{2} \phi-24 c^{3} \phi-24 s c \theta+12 c^{2} \theta+24 c^{3} \theta+8\right) \frac{\partial^{4} u}{\partial x^{4}}+O\left((\Delta x)^{4}\right)=0
\end{aligned}
$$

It is notable that the amounts of numerical diffusion are independent of the values of $s$, although the usable range of values of $c$ changes with $s$.

It can be easily seen that (2.7), (2.18), and (2.22) are special cases of (3.2).

The MEPDE which corresponds to a finite-difference method consistent with the convection-diffusion equation may be written in the general form:

$$
\frac{\partial u}{\partial t}+a \frac{\partial u}{\partial x}-\left(\alpha-\frac{a(\Delta x)}{2} d_{2}(c, s)\right) \frac{\partial^{2} u}{\partial x^{2}}+\sum_{r=3}^{\infty} \frac{a(\Delta x)^{r-1}}{r !} d_{r}(c, s) \frac{\partial^{r} u}{\partial x^{r}}=0
$$

The summed terms in (3.6) form the truncation error which indicates the order of accuracy of the corresponding finite difference equation. The MEPDE is obtained from the equivalent partial differential equation by converting all derivatives of (3.6) involving $\partial / \partial t$ except $\partial u / \partial t$, into derivatives of $x$ only. The truncation error of the MEPDE then includes only derivatives in $x$ only. There are, therefore, fewer terms of the same order to be dealt with in the MEPDE than in the equivalent partial differential equation.

A technique is called first-order accurate if it has a modified equivalent equation which has $d_{2} \neq 0$ when it is written in the form (3.6). If $d_{2}=0$ and $d_{3} \neq 0$, then the error term is $O\left((\Delta x)^{3}\right)$ and the method is said to be second-order accurate. In general, if $d_{r}=0$ for $r=2,3, \ldots, m$ and $d_{m+1} \neq 0$, then the method is said to be $m$ th-order accurate.

Using weighted differencing in order to construct higher-order procedures, weights are used to eliminate from the MEPDE as many as possible of the terms containing the derivatives $\partial^{r} u / \partial x^{r}, r=1,2, \ldots$, to develop finite difference formulas of higher orders of accuracy than conventional techniques.

For more details about MEPDE approach, its applications, and its effectiveness, see [21].

\section{Computational aspects}

A test problem is chosen to numerically validate the new discussed explicit finite difference schemes. These techniques are applied to solve (1.3)-(1.5) with $g_{0}(t), g_{1}(t)$, and $f(x)$ known and $u$ unknown. 
Table 4.1. Numerical test results for the described techniques.

\begin{tabular}{ccc|ccc}
\hline & & & Second-order & Third-order & Fourth-order \\
\hline$\Delta t$ & $\Delta x$ & $c$ & Error & Error & Error \\
\hline 0.004 & 0.02 & 0.16 & $3.6 \times 10^{-3}$ & $3.9 \times 10^{-3}$ & $2.5 \times 10^{-5}$ \\
0.008 & 0.02 & 0.32 & $3.0 \times 10^{-3}$ & $2.7 \times 10^{-3}$ & $3.3 \times 10^{-5}$ \\
0.016 & 0.02 & 0.64 & $3.8 \times 10^{-3}$ & $3.6 \times 10^{-3}$ & $1.6 \times 10^{-5}$ \\
0.008 & 0.04 & 0.16 & $2.4 \times 10^{-3}$ & $3.3 \times 10^{-3}$ & $2.9 \times 10^{-5}$ \\
0.016 & 0.04 & 0.32 & $3.6 \times 10^{-3}$ & $3.0 \times 10^{-3}$ & $3.0 \times 10^{-5}$ \\
0.032 & 0.04 & 0.64 & $3.0 \times 10^{-3}$ & $3.7 \times 10^{-3}$ & $2.7 \times 10^{-5}$ \\
0.016 & 0.08 & 0.16 & $2.8 \times 10^{-3}$ & $2.1 \times 10^{-3}$ & $3.9 \times 10^{-5}$ \\
0.032 & 0.08 & 0.32 & $4.2 \times 10^{-3}$ & $3.9 \times 10^{-3}$ & $1.8 \times 10^{-5}$ \\
0.064 & 0.08 & 0.64 & $4.0 \times 10^{-3}$ & $2.6 \times 10^{-3}$ & $2.5 \times 10^{-5}$ \\
\hline
\end{tabular}

Consider (1.3)-(1.5) with

$$
\begin{gathered}
f(x)=\exp \left[-\frac{(x-2)^{2}}{8}\right], \\
g_{0}(t)=\sqrt{\frac{20}{20+t}} \exp \left[-\frac{(5+4 t)^{2}}{10(t+20)}\right], \\
g_{1}(t)=\sqrt{\frac{20}{20+t}} \exp \left[-\frac{2(5+2 t)^{2}}{5(t+20)}\right], \\
\alpha=0.1, \\
a=0.8,
\end{gathered}
$$

for which the exact solution is

$$
u(x, t)=\sqrt{\frac{20}{20+t}} \exp \left[-\frac{(x-2-0.8 t)^{2}}{0.4(t+20)}\right] .
$$

Tests were carried out for three values of the cell Reynolds number $R_{\Delta}=c / s$, namely, $R_{\Delta}=2,4,8$. For each value of $R_{\Delta}$, three values of $c$ were used, namely, $c=0.16,0.32,0.64$. For the three tests for each $R_{\Delta}, s$ was chosen to force $\Delta t=0.004,0.008,0.016,0.032,0.064$ as the value of $c$ was increased. Equation (4.1) was used to produce values for the first time-level.

The results obtained for $u(0.25,1.0)$ computed for various values of $c$ and $s$, using the three explicit finite difference techniques described in this paper, are shown in Table 4.1.

It is worth noting that for each value of $R_{\Delta}$, three values of $\Delta x$ were used, namely, $\Delta x=$ $0.02,0.04,0.08$. For the three tests for each $R_{\Delta}, s$ was chosen to force $c=0.04,0.08,0.16$, $0.32,0.64$ as the value of $\Delta x$ was decreased. The results obtained for the second-order upwind explicit method, the third-order upwind explicit scheme, and the fourth-order upwind explicit technique are presented in Table 4.2. 
Table 4.2. Numerical test results for the described techniques.

\begin{tabular}{ccc|ccc}
\hline & & & Second-order & Third-order & Fourth-order \\
\hline$\Delta t$ & $\Delta x$ & $c$ & Error & Error & Error \\
\hline 0.004 & 0.02 & 0.16 & $3.6 \times 10^{-3}$ & $3.9 \times 10^{-3}$ & $2.5 \times 10^{-5}$ \\
0.004 & 0.04 & 0.08 & $1.0 \times 10^{-3}$ & $3.0 \times 10^{-3}$ & $4.0 \times 10^{-5}$ \\
0.004 & 0.08 & 0.04 & $4.1 \times 10^{-3}$ & $2.5 \times 10^{-3}$ & $6.5 \times 10^{-5}$ \\
0.008 & 0.02 & 0.32 & $3.0 \times 10^{-3}$ & $2.7 \times 10^{-3}$ & $3.3 \times 10^{-5}$ \\
0.008 & 0.04 & 0.16 & $2.4 \times 10^{-3}$ & $3.3 \times 10^{-3}$ & $2.9 \times 10^{-5}$ \\
0.008 & 0.08 & 0.08 & $9.5 \times 10^{-3}$ & $2.7 \times 10^{-3}$ & $4.6 \times 10^{-5}$ \\
0.016 & 0.02 & 0.64 & $3.8 \times 10^{-3}$ & $3.6 \times 10^{-3}$ & $1.6 \times 10^{-5}$ \\
0.016 & 0.04 & 0.32 & $3.6 \times 10^{-3}$ & $3.0 \times 10^{-3}$ & $3.0 \times 10^{-5}$ \\
0.016 & 0.08 & 0.16 & $2.8 \times 10^{-3}$ & $2.1 \times 10^{-3}$ & $3.9 \times 10^{-5}$ \\
\hline
\end{tabular}

Table 4.3. Numerical results at various values of $x$ at fixed time $(t=1.0)$.

\begin{tabular}{c|cccc}
\hline & Exact value & Second-order & Third-order & Fourth-order \\
\hline$x$ & & Error & Error & Error \\
\hline 0.1 & 0.4097319 & $1.3 \times 10^{-3}$ & $2.7 \times 10^{-3}$ & $3.4 \times 10^{-5}$ \\
0.2 & 0.4364170 & $1.1 \times 10^{-3}$ & $2.7 \times 10^{-3}$ & $3.2 \times 10^{-5}$ \\
0.3 & 0.4637347 & $1.2 \times 10^{-3}$ & $2.6 \times 10^{-3}$ & $3.1 \times 10^{-5}$ \\
0.4 & 0.4915904 & $1.4 \times 10^{-3}$ & $2.6 \times 10^{-3}$ & $2.9 \times 10^{-5}$ \\
0.5 & 0.5198801 & $1.3 \times 10^{-3}$ & $2.7 \times 10^{-3}$ & $2.7 \times 10^{-5}$ \\
0.6 & 0.5484904 & $1.1 \times 10^{-3}$ & $2.4 \times 10^{-3}$ & $2.7 \times 10^{-5}$ \\
0.7 & 0.5772989 & $1.4 \times 10^{-3}$ & $2.0 \times 10^{-3}$ & $2.5 \times 10^{-5}$ \\
0.8 & 0.6061756 & $1.5 \times 10^{-3}$ & $2.3 \times 10^{-3}$ & $2.2 \times 10^{-5}$ \\
0.9 & 0.6349830 & $1.7 \times 10^{-3}$ & $2.5 \times 10^{-3}$ & $2.0 \times 10^{-5}$ \\
\hline
\end{tabular}

The results obtained reflect the fourth-order convergence of the new explicit finite difference formula (2.22).

Note that the values chosen for $s$ and $c$ are in the range of the stability of all explicit finite difference schemes considered in this article.

When the results obtained for the new fourth-order explicit technique are compared with those of the second-order method, the average error of the latter is generally found to be at least two orders of magnitude larger than the former.

Inspection of Table 4.2 shows that the size of the average error obtained is closely related to the size of the dominant error term in the MEPDE of the method used.

The results obtained for the second-order upwind explicit method, the third-order upwind explicit scheme, and the fourth-order upwind explicit technique at various values of $x$ at fixed time with $c=0.24$ are given in Table 4.3.

As it can be easily seen, this table also contains the exact values. 
The CPU time required for a run with a given value of $c$ is almost independent of the value of $R_{\Delta}$ used, and this time increases with increasing $c$. In fact there was little difference in the CPU time required by the two-level explicit methods when the parameters used were the same.

Moreover, numerical methods based on the approach we used would require considerably less computational effort.

When the results obtained for the second-order explicit formula are compared with those of the third-order scheme, the average error of the former is generally found to be one order of magnitude larger than the latter.

Our attention has been confined to the constant coefficient convection-diffusion equation in one-space variable. The generalization to higher space is straightforward. In a subsequent paper we will report on the generalizations.

\section{Concluding remarks}

In this paper, various numerical methods were applied to the one-dimensional convection-diffusion equation. The discussed computational procedures solved our model quite satisfactorily. We have proposed a new practical scheme-designing approach whose application is based on the MEPDE. This approach can unify the deduction of arbitrary schemes for the solution of the convection-diffusion equation in one-space variable. This approach is especially efficient in the design of higher-order techniques. The two-level explicit finite difference schemes are very simple to implement and economical to use. They are very efficient and they need less CPU time than the implicit finite difference methods. For each of the finite difference methods investigated the MEPDE is employed which permits the order of accuracy of the numerical methods to be determined. Also from the truncation error of the modified equivalent equation, it is possible to eliminate the dominant error terms associated with the finite difference equations that contain free parameters (weights), thus leading to more accurate methods. The explicit finite difference schemes are very easy to implement for similar higher-dimensional problems, but it may be more difficult when dealing with the implicit finite difference schemes. When comparing the explicit finite difference techniques described in this report, it was found that the most accurate method is the new fourth-order explicit formula. This scheme like other explicit schemes can be used to advantage on vector or parallel computers. It is evident that the approach presented in this article can be naturally generalized to the design of finite difference methods for any linear time-dependent partial differential equation.

\section{Acknowledgment}

The author would like to thank the referee for valuable suggestions.

\section{References}

[1] P. C. Chatwin and C. M. Allen, Mathematical models of dispersion in rivers and estuaries, Annu. Rev. Fluid Mech. 17 (1985), 119-149.

[2] M. H. Chaudhry, D. E. Cass, and J. E. Edinger, Modelling of unsteady-flow water temperatures, J. Hydrol. Eng. 109 (1983), no. 5, 657-669. 


\section{Numerical solution of convection-diffusion equation}

[3] M. P. Chernesky, On preconditioned Krylov subspace methods for discrete convection-diffusion problems, Numer. Methods Partial Differential Equations 13 (1997), no. 4, 321-330.

[4] R. C. Y. Chin, T. A. Manteuffel, and J. de Pillis, ADI as a preconditioning for solving the convection-diffusion equation, SIAM J. Sci. Statist. Comput. 5 (1984), no. 2, 281-299.

[5] M. Dehghan, Quasi-implicit and two-level explicit finite-difference procedures for solving the onedimensional advection equation, to appear in Appl. Math. Comput.

[6] Q. N. Fattah and J. A. Hoopes, Dispersion in anisotropic, homogeneous, porous media, J. Hydrol. Eng. 111 (1985), no. 5, 810-827.

[7] V. Guvanasen and R. E. Volker, Numerical solutions for solute transport in unconfined aquifers, Internat. J. Numer. Methods Fluids 3 (1983), 103-123.

[8] A. C. Hindmarsh, P. M. Gresho, and D. F. Griffiths, The stability of explicit Euler time-integration for certain finite difference approximations of the multidimensional advection-diffusion equation, Internat. J. Numer. Methods Fluids 4 (1984), no. 9, 853-897.

[9] F. M. Holly Jr. and J.-M. Usseglio-Polatera, Dispersion simulation in two-dimensional tidal flow, J. Hydrol. Eng. 110 (1984), no. 7, 905-926.

[10] J. Isenberg and C. Gutfinger, Heat transfer to a draining film, Int. J. Heat Mass Transfer 16 (1973), no. 2, 505-512.

[11] N. Kumar, Unsteady flow against dispersion in finite porous media, J. Hydrol. 63 (1983), no. 3-4, 345-358.

[12] L. Lapidus and N. R. Amundson, Mathematics of absorption in beds. VI. The effect of longitudinal diffusion in ion-exchange and chromatographic columns, J. Phys. Chem. 56 (1952), no. 8, 984-988.

[13] P. D. Lax and B. Wendroff, Difference schemes for hyperbolic equations with high order of accuracy, Comm. Pure Appl. Math. 17 (1964), 381-398.

[14] D. Liang and W. Zhao, A high-order upwind method for the convection-diffusion problem, Comput. Methods Appl. Mech. Engrg. 147 (1997), no. 1-2, 105-115.

[15] A. R. Mitchell and D. F. Griffiths, The Finite Difference Method in Partial Differential Equations, John Wiley \& Sons, Chichester, 1980.

[16] J. Noye, Numerical solutions of partial differential equations, Lecture Notes, 1989.

[17] J.-Y. Parlange, Water transport in soils, Annu. Rev. Fluid Mech. 12 (1980), 77-102.

[18] J. R. Salmon, J. A. Liggett, and R. H. Gallagher, Dispersion analysis in homogeneous lakes, Internat. J. Numer. Methods Engrg. 15 (1980), no. 11, 1627-1642.

[19] S.-C. Sheen and J.-L. Wu, Preconditioning techniques for the BiCGSTAB algorithm used in convection-diffusion problems, Numer. Heat Transfer, Part B 34 (1998), 241-256.

[20] J. C. Strikwerda, Finite Difference Schemes and Partial Differential Equations, The Wadsworth \& Brooks/Cole Mathematics Series, Wadsworth \& Brooks/Cole Advanced Books \& Software, California, 1989.

[21] R. F. Warming and B. J. Hyett, The modified equation approach to the stability and accuracy analysis of finite-difference methods, J. Comput. Phys. 14 (1974), no. 2, 159-179.

[22] Z. Zlatev, R. Berkowicz, and L. P. Prahm, Implementation of a variable stepsize variable formula method in the time-integration part of a code for treatment of long-range transport of air pollutants, J. Comput. Phys. 55 (1984), no. 2, 278-301.

Mehdi Dehghan: Department of Applied Mathematics, Faculty of Mathematics and Computer Science, Amirkabir University of Technology, Tehran 15914, Iran

E-mail address: mdehghan@aut.ac.ir 


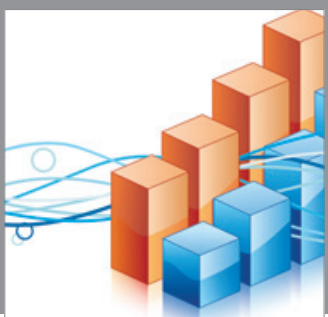

Advances in

Operations Research

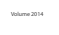

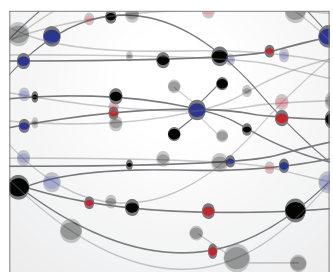

\section{The Scientific} World Journal
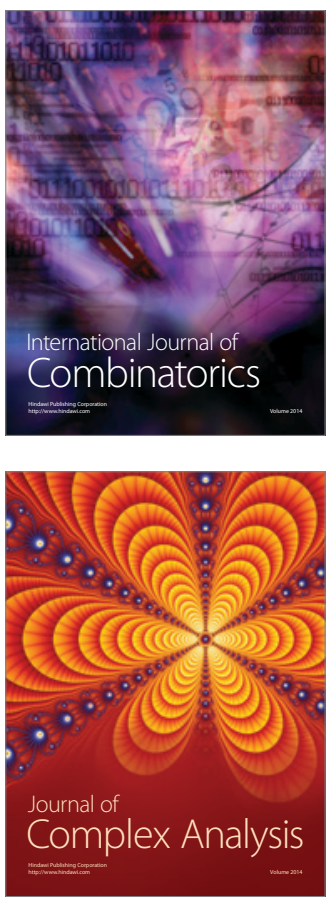

International Journal of

Mathematics and

Mathematical

Sciences
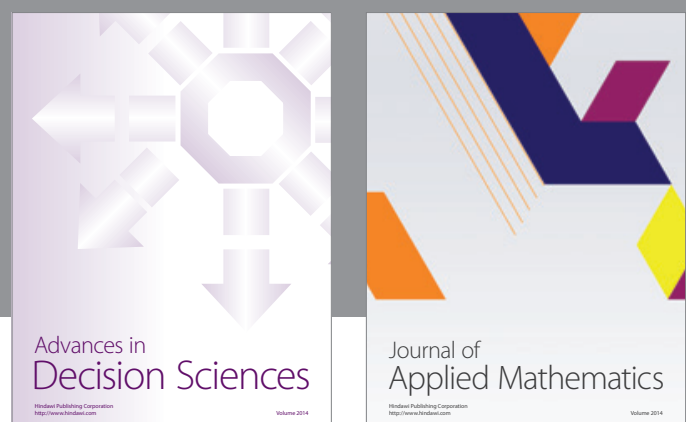

Journal of

Applied Mathematics
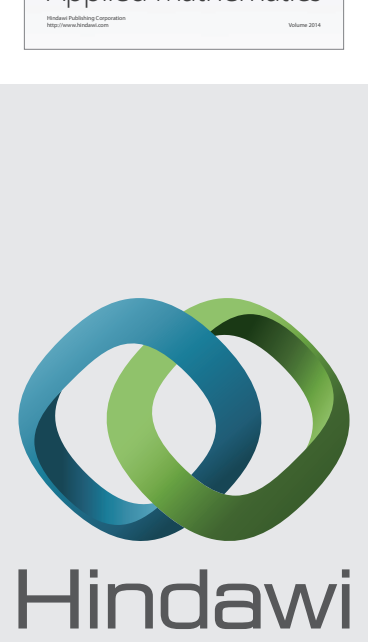

Submit your manuscripts at http://www.hindawi.com
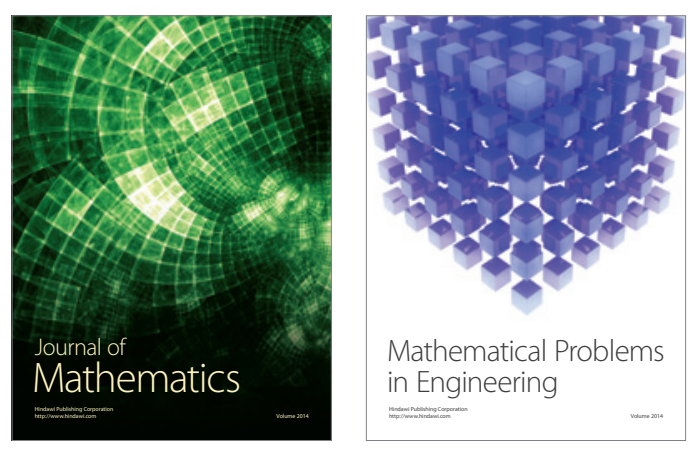

Mathematical Problems in Engineering
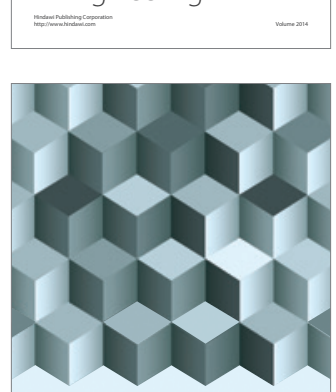

Journal of

Function Spaces
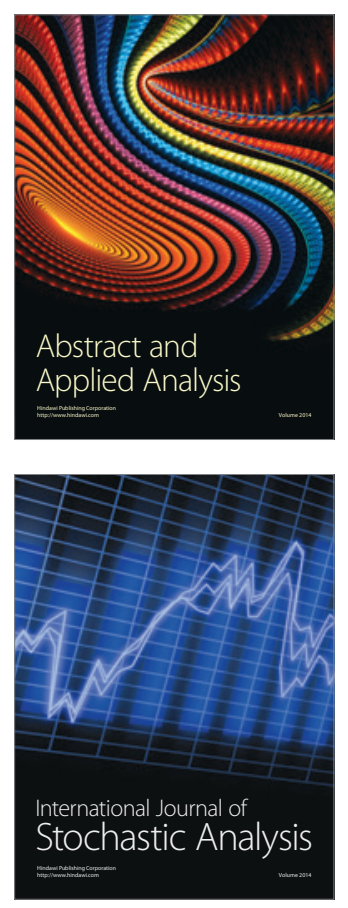

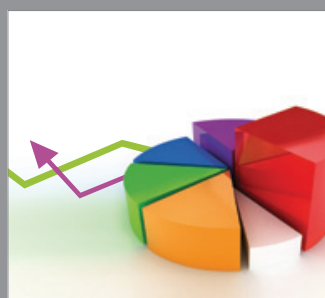

ournal of

Probability and Statistics

Promensencen
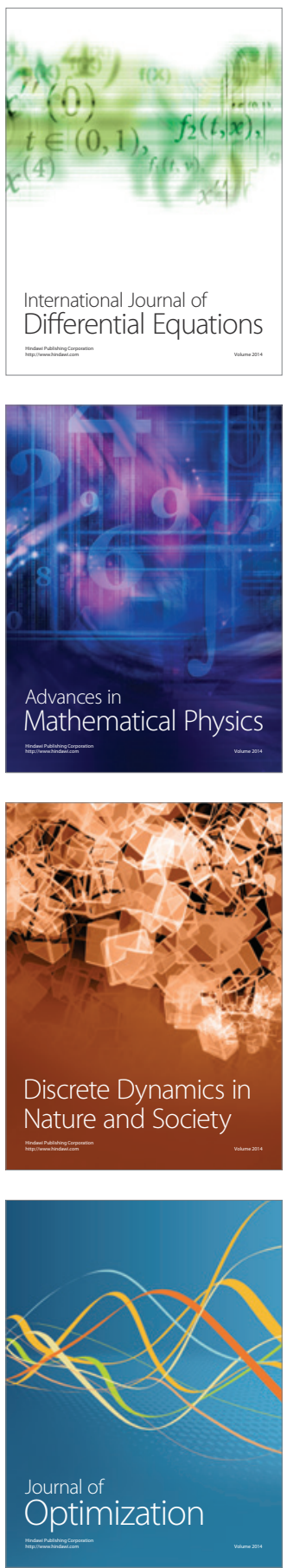